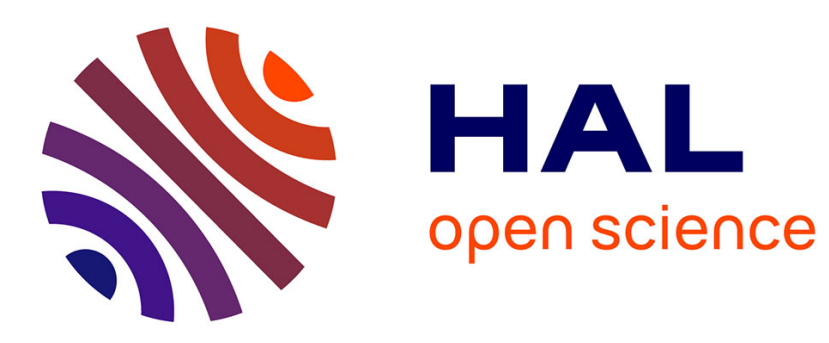

\title{
Improving 3D MA-TIRF Reconstruction with Deconvolution and Background Estimation
}

Emmanuel Soubies, Laure Blanc-Féraud, Sébastien Schaub, Ellen van Obberghen-Schilling

\section{- To cite this version:}

Emmanuel Soubies, Laure Blanc-Féraud, Sébastien Schaub, Ellen van Obberghen-Schilling. Improving 3D MA-TIRF Reconstruction with Deconvolution and Background Estimation. IEEE International Symposium on Biomedical Imaging, Apr 2019, Venise, Italy. 10.1109/ISBI.2019.8759103 . hal-02017862

\section{HAL Id: hal-02017862 \\ https://hal.inria.fr/hal-02017862}

Submitted on 13 Feb 2019

HAL is a multi-disciplinary open access archive for the deposit and dissemination of scientific research documents, whether they are published or not. The documents may come from teaching and research institutions in France or abroad, or from public or private research centers.
L'archive ouverte pluridisciplinaire HAL, est destinée au dépôt et à la diffusion de documents scientifiques de niveau recherche, publiés ou non, émanant des établissements d'enseignement et de recherche français ou étrangers, des laboratoires publics ou privés. 


\title{
IMPROVING 3D MA-TIRF RECONSTRUCTION WITH DECONVOLUTION AND BACKGROUND ESTIMATION
}

\author{
Emmanuel Soubies ${ }^{1}$, Laure Blanc-Féraud ${ }^{2}$, Sébastien Schaub ${ }^{3}$, and Ellen Van Obberghen-Schilling ${ }^{3}$ \\ ${ }^{1}$ Biomedical Imaging Group, EPFL, Lausanne, Switzerland \\ ${ }^{2}$ Université Côte d'Azur, CNRS, Inria, I3S, France \\ ${ }^{3}$ Université Côte d'Azur, CNRS, Inserm, iBV, France
}

\begin{abstract}
Total internal reflection fluorescence microscopy (TIRF) produces 2D images of the fluorescent activity integrated over a very thin layer adjacent to the glass coverslip. By varying the illumination angle (multi-angle TIRF), a stack of 2D images is acquired from which it is possible to estimate the axial position of the observed biological structures. Due to its unique optical sectioning capability, this technique is ideal to observe and study biological processes at the vicinity of the cell membrane. In this paper, we propose an efficient reconstruction algorithm for multi-angle TIRF microscopy which accounts for both the PSF of the acquisition system (diffraction) and the background signal (e.g., autofluorescence). It jointly performs volume reconstruction, deconvolution, and background estimation. This algorithm, based on the simultaneous-direction method of multipliers (SDMM), relies on a suitable splitting of the optimization problem which allows to obtain closed form solutions at each step of the algorithm. Finally, numerical experiments reveal the importance of considering the background signal into the reconstruction process, which reinforces the relevance of the proposed approach.
\end{abstract}

Index Terms - Total-Internal Reflection Fluorescence Microscopy, Inverse Problems, SDMM

\section{INTRODUCTION}

Due to its capability to limit the observed region to a thin layer of a few hundred nanometers (below 100nm up to $1 \mu \mathrm{m}$ ), total internal reflection fluorescence (TIRF) microscopy [1, 2, 3, 4] is ideally suited to the observation of biological activities near the cell membrane. This unique optical sectioning is made possible by using an evanescent excitation produced in the total internal reflection regime. Moreover, given a set of TIRF acquisitions with different incident angles (multi-angle TIRF, MA-TIRF) it is possible to reconstruct a $3 \mathrm{D}$ volume through dedicated algorithms $[5,6,7,8,9,10,11,12,13$, $14,15,16,17,18,19]$. This gives access to a precise axial localization of the molecules within the observed layer, with a precision of the order of $30 \mathrm{~nm}$. To further improve the quality of the reconstruction, it is important to consider both the PSF of the system as well as a background signal which corrupt the acquired data. In a prior work [20], we proposed a joint reconstruction and deconvolution algorithm for MA-TIRF microscopy. However, the background sig-

E.S. work was funded by an ERC grant (ERC-692726-GlobalBioIm). Work on TIRF was supported by the French Government (National Research Agency) through the Investments for the Future LABEX SIGNALIFE (ANR11- LABX-0028-01) and the ANR-FNS Collaborative Research Programme (ANR-16-CE93-0005-01).

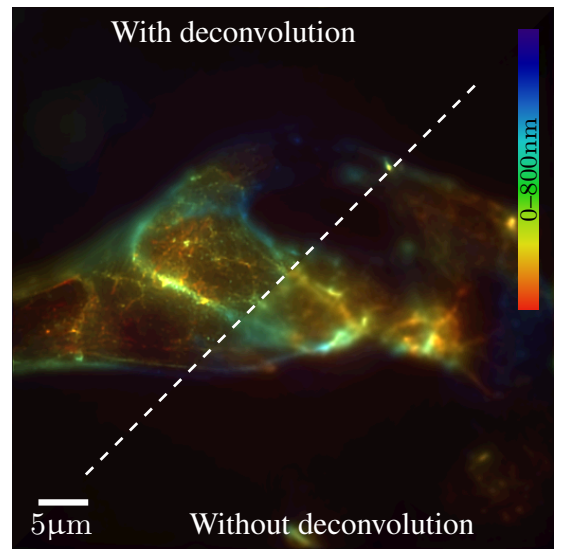

Fig. 1: MA-TIRF reconstruction (proposed method) of actin filaments. Results with and without considering the convolution operator in the model are presented. The colormap encodes the axial position of the reconstructed structures from 0 to $800 \mathrm{~nm}$.

nal is estimated and subtracted from the acquired data within a preprocessing step, as proposed by the authors in [15]. In the present communication, we extend the algorithm in [20] to jointly perform $3 \mathrm{D}$ reconstruction, deconvolution, and background estimation. A reconstruction obtained with the proposed method is presented in Figure 1. Moreover, we analyze the effect of the background signal on the reconstructed volume and show that it is crucial to estimate it in order to produce relevant reconstructions.

\section{IMAGE FORMATION MODEL}

A TIRF acquisition (2D) $g \in \mathbb{R}^{M}$ follows the generic fluorescent microscopy model

$$
g_{m}=(h *(w f))\left(\mathbf{x}_{m}, z_{\mathrm{fp}}\right)+b_{m}
$$

where $g_{m}$ is the $m$ th component of the discrete vector $\mathbf{g}, w \in$ $L_{2}\left(\mathbb{R}^{3}\right)$ is the illumination, $h \in L_{2}\left(\mathbb{R}^{3}\right)$ denotes the point-spread function (PSF) (including the integration over the $m$ th pixel), and $f \in L_{2}\left(\mathbb{R}^{3}\right)$ is the unknown 3D fluorophores density map. Model (1) maps the continuous object $f$ to the discrete measurements g. This is achieved by sampling the continuous quantity $(h *(w f))$ at the lateral positions $\left\{\mathbf{x}_{m} \in \mathbb{R}^{2}\right\}_{m=1}^{M}$ of the camera pixels and the axial position $z_{\mathrm{fp}}$ of the objective focal plane. Fi- 
nally $\mathbf{b} \in \mathbb{R}^{M}$ is a vector modeling the background signal (e.g., auto-fluorescence) and noise (discrete setting).

The specificity of TIRF microscopy lies in the illumination process. It proceeds by illuminating the sample with an incident angle greater than the critical angle of reflection, so that the light is totally reflected by the coverslip (total internal reflection regime). This phenomenon produces the evanescent excitation $[1,2,4]$

$$
w(\mathbf{x}, z)=\tilde{w}(z)=w_{0} \exp (-z p),
$$

for $z>0$, where $w_{0}$ is the intensity at the interface (i.e., $z=0$ ) and $p$ characterizes the inverse of the penetration depth of the evanescent wave. It has the expression

$$
p=\frac{4 \pi n_{i}}{\lambda_{\text {exc }}}\left(\sin ^{2}(\alpha)-\sin ^{2}\left(\alpha_{c}\right)\right),
$$

where $n_{i}$ ( $n_{t}$, respectively) is the refractive index of the incident medium (the transmitted medium, respectively), $\lambda_{\mathrm{exc}}$ is the wavelength of the incident beam, $\alpha_{c}=\operatorname{asin}\left(n_{t} / n_{i}\right)$ is the critical angle from which there is total internal reflection of the light, and $\alpha>\alpha_{c}$ is the incident angle of the illumination beam. The intensity $w_{0}$ depends on the angle $\alpha$ as well as the polarization of the incident beam and is assumed spatially constant. A theoretical expression can be derived from Fresnel's equations $[4,15]$.

Because of the fast decay of the excitation field (2) in depth (with respect to the $z$ variable), only a thin layer of the sample at the vicinity of the glass coverslip is imaged (between $100 \mathrm{~nm}$ to $1 \mu \mathrm{m}$, depending on the incident angle). Within this thin region, the variations of the PSF in depth are small and we can thus assume that $h$ is constant along $z$ (i.e., $h(\mathbf{x}, z)=\tilde{h}(\mathbf{x}))$. This allows to decouple the spatial convolution and the TIRF excitation in Model (1),

$$
\begin{aligned}
g_{m} & =\int_{0}^{\infty} \int_{\mathbb{R}^{2}} \tilde{h}\left(\mathbf{x}_{m}-\mathbf{x}\right) \tilde{w}(z) f(\mathbf{x}, z) \mathrm{d} \mathbf{x} \mathrm{d} z+b_{m} \\
& =\int_{0}^{\infty} \tilde{w}(z) \int_{\mathbb{R}^{2}} \tilde{h}\left(\mathbf{x}_{m}-\mathbf{x}\right) f(\mathbf{x}, z) \mathrm{d} \mathbf{x} \mathrm{d} z+b_{m}
\end{aligned}
$$

which is the key ingredient to derive the SDMM that we propose in Section 3.

A single TIRF image does not provide a quantitative information on the location of the fluorescent probes within the observed layer. However, given a set of $Q$ TIRF images $\left\{\mathbf{g}_{q} \in \mathbb{R}^{M}\right\}_{q=1}^{Q}$ acquired with the incident angles $\left\{\alpha_{q}>\alpha_{c}\right\}_{q=1}^{Q}$, it is possible to numerically reconstruct the $3 \mathrm{D}$ biological sample $f$. To that end, we define the discrete version of Model (4) for the incident angle $\alpha_{q}$ as

$$
\mathbf{g}_{q}=\mathbf{T}_{q} \mathbf{H} \mathbf{f}+\mathbf{b}
$$

where $\mathbf{f} \in \mathbb{R}_{\geqslant 0}^{N}$ denotes the 3D discrete fluorophore density map, $\mathbf{H} \in \mathbb{R}^{N \times N}$ is a convolution operator that convolves each z-slice of the volume $\mathbf{f}$ with a sampled version of the $2 \mathrm{D}$ kernel $\tilde{h}$, and $\mathbf{T}_{q} \in \mathbb{R}^{N \times M}$ is the discrete TIRF operator associated to the incident angle $\alpha_{q}$. It performs a weighted sum of the slices of $\mathbf{H f}$, where the weights are samples of $\tilde{w}$ associated to $\alpha_{q}$. Note that, in contrast, the background signal $\mathbf{b}$ is considered independent from the incident angle (i.e., independent of the TIRF excitation process).

\section{JOINT DECONVOLUTION, RECONSTRUCTION AND BACKGROUND ESTIMATION}

We aim at estimating both the 3D fluorophore density $\mathbf{f}$ and the (2D) background signal $\mathbf{b}$. We formulate the reconstruction problem as the joint optimization problem

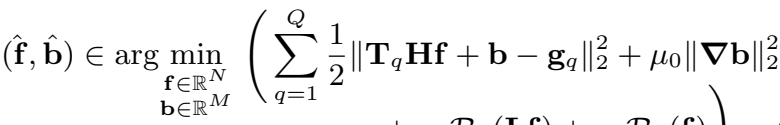

$$
\begin{aligned}
& \left.+\mu_{1} \mathcal{R}_{1}(\mathbf{L f})+\mu_{2} \mathcal{R}_{2}(\mathbf{f})\right) .
\end{aligned}
$$

Because the background has smooth variations, we consider the first order Tikhonov regularizer $\|\nabla \mathbf{b}\|_{2}^{2}$ for $\mathbf{b}$. For $\mathbf{f}$, the problem is regularized using the combination of two terms: $\mathcal{R}_{1}(\mathbf{L} \cdot)$ and $\mathcal{R}_{2}(\cdot)$. The only requirement we make is that $\mathcal{R}_{1}$ and $\mathcal{R}_{2}$ are simple in the sense that their proximal operators can be computed efficiently. In this work, we will consider the Hessian Schatten-norm of order one [21], defined by $\mathcal{R}_{1}(\mathbf{L} \cdot)=\left\|\mathbf{H}_{\mathbf{e}} \cdot\right\|_{\star, 1}$, as well as the sum of a nonnegativity constraint and a sparsity promoting functional $\mathcal{R}_{2}=i_{\geq 0}+\|\cdot\|_{1}$, where $i_{\geq 0}(\mathbf{f})=\left\{0\right.$ if $\mathbf{f} \in \mathbb{R}_{\geq 0}^{N} ;+\infty$ otherwise $\}$. It is noteworthy that the proposed approach can also deal with the popular total variation (TV) [22] regularizer (i.e., $\mathcal{R}_{1}=\|\cdot\|_{2,1}$ and $\mathbf{L}=\boldsymbol{\nabla})$.

One can easily check that the objective function in (6), with the regularizers considered in this work, is convex in $(\mathbf{f}, \mathbf{b})$. Hence, we benefit from the variety of existing convex optimization algorithms to tackle this minimization problem. We deploy the $\mathrm{SDMM}^{1}$ [23] to solve the augmented problem

$$
\begin{aligned}
\left(\hat{\mathbf{f}}, \hat{\mathbf{b}},\left\{\hat{\mathbf{u}}_{i}\right\}_{i=0}^{2}\right) \in \underset{\substack{\left\{, \mathbf{b} \\
\left\{\hat{\mathbf{u}}_{i}\right\}_{i=0}^{2}\right.}}{\arg \min }\left(\sum_{q=1}^{Q} \frac{1}{2}\left\|\mathbf{T}_{q} \mathbf{u}_{0}+\mathbf{b}-\mathbf{g}_{q}\right\|_{2}^{2}+\mu_{0}\|\nabla \mathbf{b}\|_{2}^{2}\right. \\
\left.+\mu_{1} \mathcal{R}_{1}\left(\mathbf{u}_{1}\right)+\mu_{2} \mathcal{R}_{2}\left(\mathbf{u}_{2}\right)\right) \\
\text { such that } \quad \mathbf{u}_{0}=\mathbf{H f}, \mathbf{u}_{1}=\mathbf{L f}, \mathbf{u}_{2}=\mathbf{f} .
\end{aligned}
$$

The iterates are summarized in Algorithm 1. The parameters $\left\{\rho_{i}\right\}_{i=0}^{2}$ are the Lagrangian multipliers that act as preconditioners. In practice, we tune them empirically to optimize the convergence speed. Lines 7 to 9 require to compute different proximity operators. For $\mathcal{R}_{1}=\|\cdot\|_{*, 1}$, it admits a closed form expression which can be efficiently computed through singular value decomposition [21]. The proximal operator of $\mathcal{R}_{2}=i_{\geq 0}+\|\cdot\|_{1}$ has a very simple component-wise expression given by

$$
\left[\operatorname{prox}_{\frac{\mu_{2}}{\rho_{2}} \mathcal{R}_{2}}(\mathbf{v})\right]_{n}=\max \left(0, v_{n}-\mu_{2} / \rho_{2}\right) .
$$

Finally, for $\mathcal{D}=\frac{1}{2 \rho_{0}} \sum_{q=1}^{Q}\left\|\mathbf{T}_{q} \cdot+\mathbf{b}^{k}-\mathbf{g}_{q}\right\|_{2}^{2}$, we have

$$
\operatorname{prox}_{\mathcal{D}}(\mathbf{v})=\left(\sum_{q=1}^{Q} \mathbf{T}_{q}^{T} \mathbf{T}_{q}+\rho_{0} \mathbf{I}\right)^{-1}\left(\sum_{q=1}^{Q} \mathbf{T}_{q}^{T}\left(\mathbf{g}_{q}-\mathbf{b}^{k}\right)+\rho_{0} \mathbf{v}\right)
$$

Because the TIRF excitation (2) is independant from the lateral variable $\mathbf{x}$, the operator $\sum_{q=1}^{Q} \mathbf{T}_{q}^{T} \mathbf{T}_{q}$ is block diagonal with identical blocks $\mathbf{B} \in \mathbb{R}^{N_{z} \times N_{z}}$ defined by

$$
[\mathbf{B}]_{i j}=\sum_{q=1}^{Q} w_{0, q}^{2} \exp \left(-z_{i} p_{q}\right) \exp \left(-z_{j} p_{q}\right)
$$

where $\left\{z_{i}\right\}_{i=1}^{N_{z}}$ are the axial discretization points and $p_{q}\left(w_{0, q}\right.$, respectively) is given by (3) ((2), respectively) with $\alpha=\alpha_{q}$. Hence

\footnotetext{
${ }^{1}$ Extension of the popular alternating direction method of multipliers (ADMM) to minimize the sum of more than two functionals.
} 


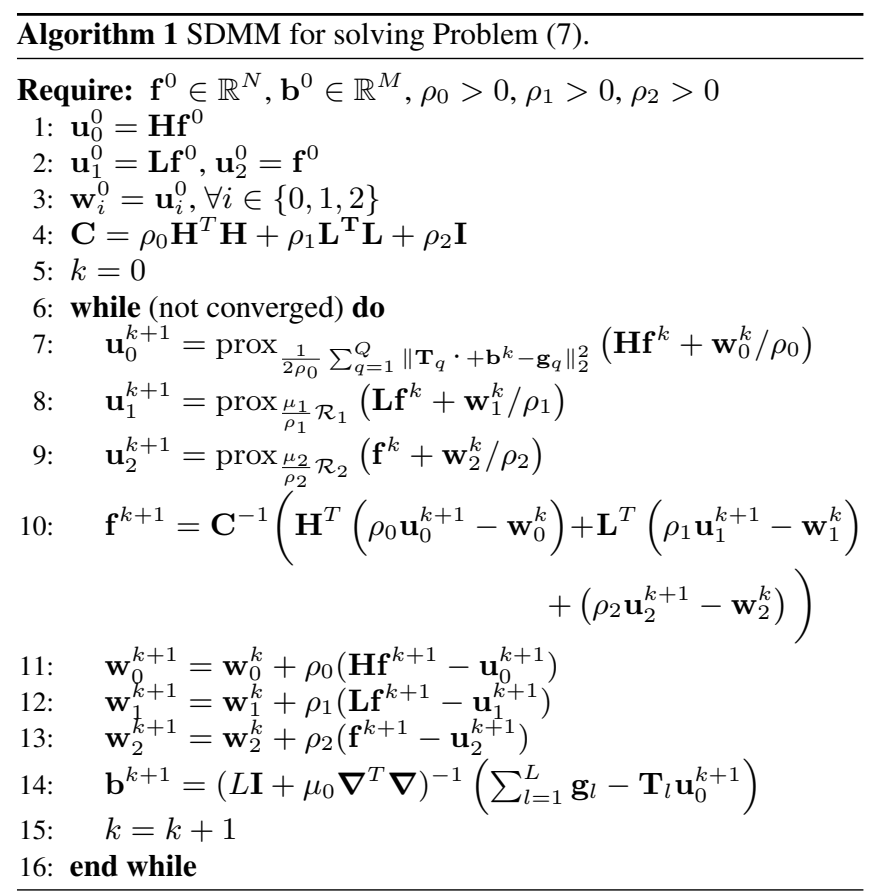

$\left(\sum_{q=1}^{Q} \mathbf{T}_{q}^{T} \mathbf{T}_{q}+\rho_{0} \mathbf{I}\right)$ is also block diagonal and its inversion requires to compute once and store the inverse of the small size bloc $\left(\mathbf{B}+\rho_{0} \mathbf{I}\right)$ (typically smaller than $50 \times 50$ ).

Regarding the linear steps in Lines 10 and 14, they can be easily solved in the Fourier domain. Indeed, considering periodic boundary conditions, both $\mathbf{H}_{\mathbf{e}}{ }^{T} \mathbf{H}_{\mathbf{e}}$ (i.e., $\mathbf{L}^{T} \mathbf{L}$ in the present paper) and $\boldsymbol{\nabla}^{T} \boldsymbol{\nabla}$ are convolution operators. Hence, $\left(\rho_{0} \mathbf{H}^{T} \mathbf{H}+\rho_{1} \mathbf{L}^{\mathbf{T}} \mathbf{L}+\rho_{2} \mathbf{I}\right)$ and $\left(L \mathbf{I}+\mu_{0} \boldsymbol{\nabla}^{T} \boldsymbol{\nabla}\right)$ are also convolution operators which can be inverted in the Fourier domain.

\section{IMPORTANCE OF BACKGROUND ESTIMATION}

In this section we study the influence of the background signal on the reconstructed volume. Our main result is stated in Proposition 4.1.

Proposition 4.1. Let $\mathcal{T}: L_{2}(\mathbb{R}) \rightarrow \mathbb{R}$ be the $1 D$ TIRF operator such that $\mathcal{T}\{f\}(\alpha)=w_{0}(\alpha) \int_{0}^{\infty} f(z) \exp (-z p(\alpha)) \mathrm{d} z$. Let $\mathcal{A}=$ $\left\{\alpha_{q}>\alpha_{c}\right\}_{q=1}^{Q}$ be a set of incident angles and $\mathbf{g} \in \mathbb{R}^{Q}$ be the constant vector such that $g_{q}=b_{g}>0$ for all $q \in\{1, \ldots, Q\}$. Then, the solution of the optimization problem

$$
f^{\star}=\arg \min _{f \geq 0} \frac{1}{2} \sum_{q=1}^{Q}\left(\mathcal{T}\{f\}\left(\alpha_{q}\right)-g_{q}\right)^{2}
$$

is given by

$$
f^{\star}=\left(\frac{\sum_{q=1}^{Q} w_{0}\left(\alpha_{q}\right)}{\sum_{q=1}^{Q} w_{0}^{2}\left(\alpha_{q}\right)} b_{g}\right) \delta
$$

where $\delta$ denotes the Dirac impulse.

Proof. The proof can be found in [24, Proposition 3.1].

From Proposition 4.1, we get that the best interpretation (in the sense of positive least-squares) of a constant signal by the TIRF model (i.e., (4) without the convolution operator) is a Dirac mass (x,y) color-coded depth proj.

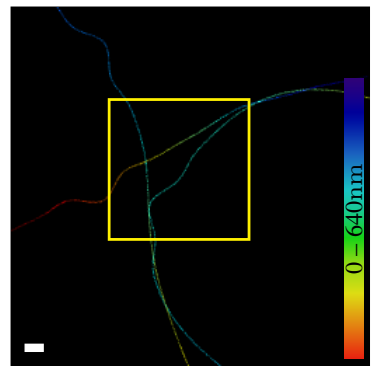

Zoom: (x,y) color-coded depth proj.
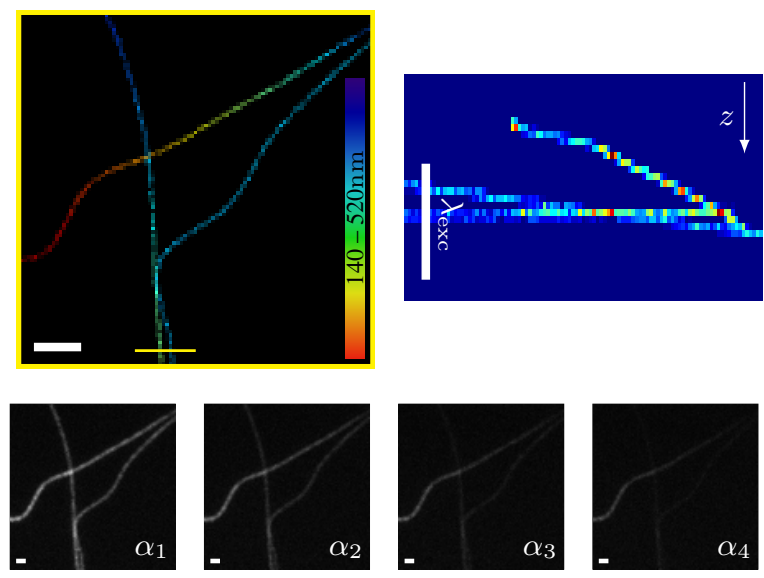

Fig. 2: Simulated data. Top row: color-coded depth representation of the ground-truth (left) and background signal (right). Middle row: zoom corresponding to the yellow square (left) and the corresponding (y,z) projection (average intensity). The yellow line in the (x,y) zoom is the one used for the profiles that are displayed in the bottom graph of Figure 3. Bottom row: examples of TIRF acquisitions for $\alpha_{c}<\alpha_{1}<\alpha_{2}<\alpha_{3}<\alpha_{4}<\alpha_{\max }$. When not specified the scale bar represents $2 \lambda_{\text {exc }}$.

localized at $z=0$. It is noteworthy to mention that the TIRF operator (i.e., the first integral in (4)) only depends on $z$, and that the result of Proposition 4.1 is thus applicable in 3D. In practice, a TIRF acquisition contains the signal resulting from the excitation of the sample plus a constant signal (background). Hence, trying to reconstruct the 3D sample from such data without considering the background signal will produce a strong signal at $z=0$ and will introduce errors in the axial localization of the fluorescent probes.

One solution to this problem, adopted by Boulanger et al [15], consists in estimating the background signal using an additional "dark image" and subtracting this background value to the acquired data before reconstruction. Note that, to account for the uncertainty of this estimation, the authors in [15] relax the nonnegativity constraint $i_{\geq 0}$ by the distance to the set of nonnegative vectors. In contrast, we propose in this work to estimate the background signal jointly with the reconstruction of the fluorophore density map.

\section{NUMERICAL EXPERIMENTS}

Simulation Settings We consider the synthetic sample depicted in Figure 2 (top left). It has been obtained from the list of molecules positions of the MT0 dataset created for the SMLM challenge [25]. More precisely, these molecules have been binned into a 3D discrete 

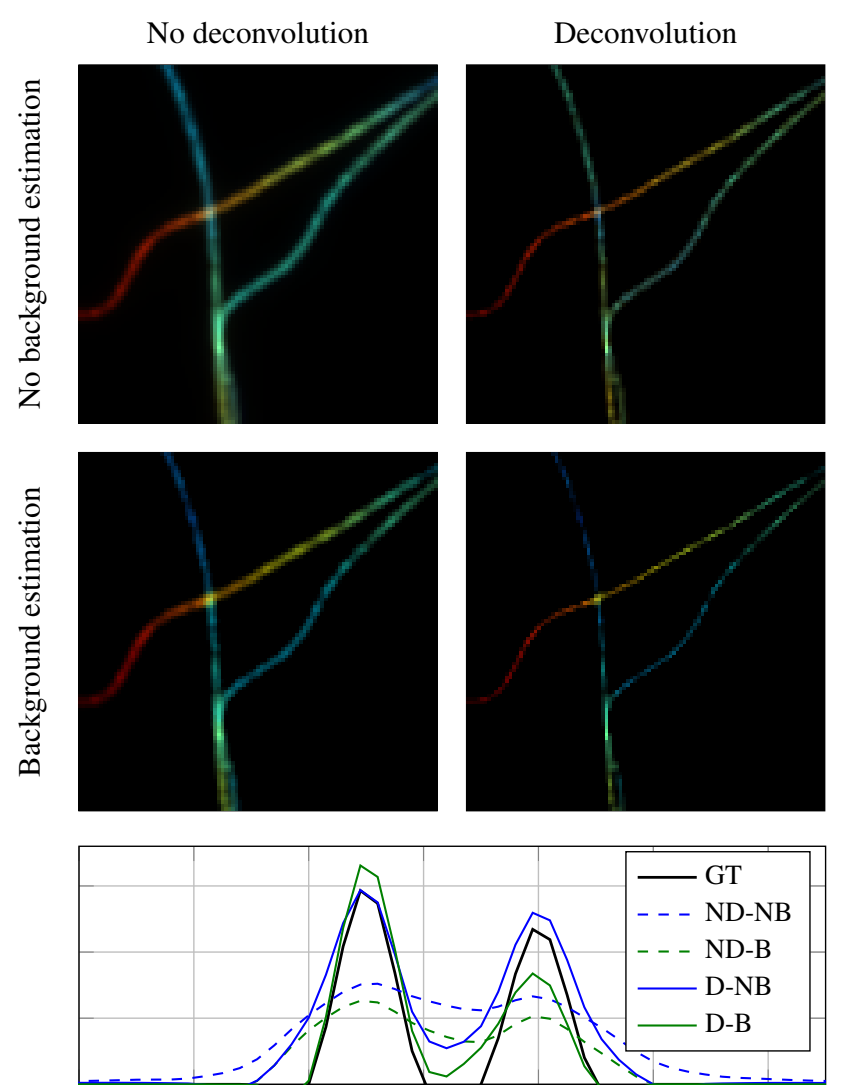

Fig. 3: Color-coded depth representations of the reconstructed volumes within the yellow region in the top-left image of Figure 2. The colormap is the one displayed in Figure 2. Profiles of the $(\mathrm{x}, \mathrm{y})$ projections (average intensity) along the yellow line drawn in Figure 2.

volume with voxel size $100 \mathrm{~nm} \times 100 \mathrm{~nm} \times 20 \mathrm{~nm}$ by considering 200 photons per molecule. In the following, we use this volume as the ground-truth reference. However, we generated the MA-TIRF acquisitions using directly the list of molecules (and not the discrete ground-truth reference). We proceeded by adding iteratively the contribution of each molecule to the acquisitions by considering their continuous positions and 200 photons per molecule. Hence, the acquisitions are generated from the continuous model (1) which prevents the so-called inverse crime. Acquisition parameters have been fixed to $n_{i}=1.518, n_{t}=1.34, \lambda_{\text {exc }}=640 \mathrm{~nm}$ and 13 incident angles (i.e., $Q=13$ ) uniformly distributed between the critical angle $\alpha_{c}$ and the maximal angle $\alpha_{\max }=\operatorname{asin}\left(\mathrm{NA} / n_{i}\right)$ that one can reach with an objective based TIRF (the numerical aperture is fixed to NA = 1.33). We considered the Born-and-Wolf PSF model. Finally, a background with values between 15 and 20 photons has been added to the data (Figure 2, top right) and the resulting noiseless acquisitions have been corrupted by a Poisson noise. Examples of acquired data are presented in Figure 2 (bottom line).

Reconstruction Results and Discussion From these simulated data, we performed several reconstructions by considering the background estimation (B) or not (NB), as well as the deconvolution (D) or not (ND). The reconstruction algorithm has been implemented using the GlobalBioIm Library [26]. Regularization parameters have been empirically tuned so as to maximize the performances.
No background estimation

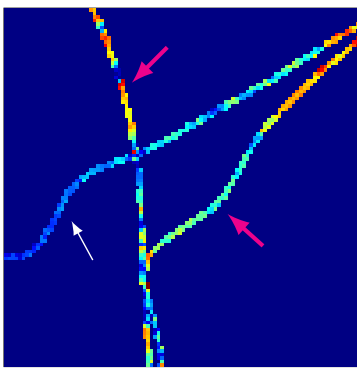

\section{Background estimation}

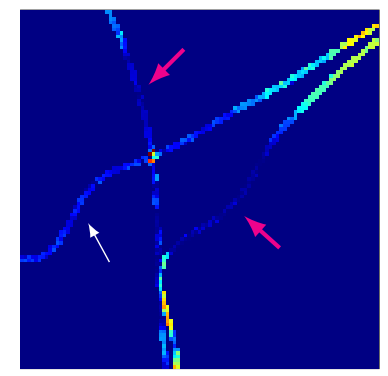

\section{$0-100 \mathrm{~nm}$}

Fig. 4: Mean axial error maps for the reconstructed volumes with deconvolution.
No background estimation

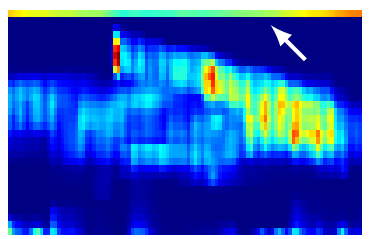

Background estimation

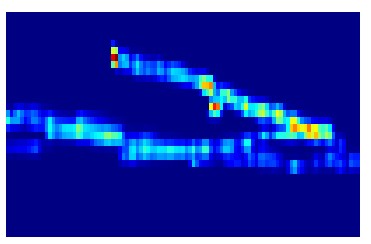

Fig. 5: (y,z) projection (average intensity) for the reconstructed volumes with deconvolution.

Color-coded depth maps are depicted in Figure 3. One can clearly observe the improvement obtained when the deconvolution is activated, in particular with the line profiles presented in the bottom graph of Figure 3. Then, in order to analyze the effect of the background estimation on the reconstructed volume, we have computed mean axial error maps between the reconstructed volumes and the ground-truth. They are provided in Figure 4. For structures that are close to the glass coverslip (i.e., $z=0$ ), the mean axial error is reasonably low, even without background estimation (thin white arrow). However, for deeper structures, estimating the background signal results in reconstructions which are more accurate (thick magenta arrows). These observations are corroborated with the $(y, z)$ projections depicted in Figure 5. These projections reveal that the axial localization of the observed structures is significantly improved when the background signal is estimated. Moreover, when the background signal is not estimated, the reconstructed volume presents a strong signal at the interface (white arrow), which illustrates the statement of Proposition 4.1. Finally, an example of reconstruction with real data is presented in Figure 1. Here also, one can appreciate the effect of the deconvolution. We refer the reader to the companion paper [20] for further experiments on real data.

\section{CONCLUSION}

We proposed an efficient reconstruction algorithm for MA-TIRF microscopy which performs at the same time reconstruction, deconvolution, and background estimation. Moreover, we demonstrated that it is crucial to estimate the background signal present in the TIRF acquisitions in order to produce relevant reconstructions. 


\section{REFERENCES}

[1] Daniel Axelrod, "Cell-substrate contacts illuminated by total internal reflection fluorescence.," The Journal of cell biology, vol. 89, no. 1, pp. 141-145, 1981.

[2] Daniel Axelrod, "Total internal reflection fluorescence microscopy," Methods in cell biology, vol. 89, pp. 169-221, 2008.

[3] Natalie S Poulter, William TE Pitkeathly, Philip J Smith, and Joshua Z Rappoport, "The physical basis of total internal reflection fluorescence (TIRF) microscopy and its cellular applications," in Advanced Fluorescence Microscopy, pp. 1-23. Springer, 2015.

[4] ML Martin-Fernandez, CJ Tynan, and SED Webb, "A "pocket guide" to total internal reflection fluorescence," Journal of microscopy, vol. 252, no. 1, pp. 16-22, 2013.

[5] WM Reichert and GA Truskey, "Total internal reflection fluorescence (tirf) microscopy. i. modelling cell contact region fluorescence," Journal of cell science, vol. 96, no. 2, pp. 219-230, 1990.

[6] Jeffrey S Burmeister, George A Truskey, and William M Reichert, "Quantitative analysis of variable-angle total internal reflection fluorescence microscopy (va-tirfm) of cell/substrate contacts," Journal of microscopy, vol. 173, no. 1, pp. 39-51, 1994.

[7] Bence P Olveczky, N Periasamy, and AS Verkman, "Mapping fluorophore distributions in three dimensions by quantitative multiple angle-total internal reflection fluorescence microscopy," Biophysical journal, vol. 73, no. 5, pp. 2836-2847, 1997.

[8] Alexander Rohrbach, "Observing secretory granules with a multiangle evanescent wave microscope," Biophysical journal, vol. 78, no. 5, pp. 2641-2654, 2000.

[9] Dinah Loerke, Walter Stühmer, and Martin Oheim, "Quantifying axial secretory-granule motion with variable-angle evanescent-field excitation," Journal of neuroscience methods, vol. 119, no. 1, pp. 65-73, 2002.

[10] Saveez Saffarian and Tomas Kirchhausen, "Differential evanescence nanometry: live-cell fluorescence measurements with 10-nm axial resolution on the plasma membrane," Biophysical journal, vol. 94, no. 6, pp. 2333-2342, 2008.

[11] Liang Liang, Hongying Shen, Yingke Xu, Pietro De Camilli, Derek K Toomre, and James S Duncan, "A Bayesian method for 3D estimation of subcellular particle features in multi-angle tirf microscopy," in Biomedical Imaging (ISBI), 2012 9th IEEE International Symposium on. IEEE, 2012, pp. 984-987.

[12] Qian Yang, Alexander Karpikov, Derek Toomre, and James S Duncan, "3-d reconstruction of microtubules from multi-angle total internal reflection fluorescence microscopy using bayesian framework," IEEE transactions on Image Processing, vol. 20, no. 8, pp. 2248-2259, 2011.

[13] Marcelina Cardoso Dos Santos, Régis Déturche, Cyrille Vézy, and Rodolphe Jaffiol, "Axial nanoscale localization by normalized total internal reflection fluorescence microscopy," Optics letters, vol. 39, no. 4, pp. 869-872, 2014.

[14] Emmanuel Soubies, Laure Blanc-Féraud, Sébastien Schaub, and Gilles Aubert, "A 3D model with shape prior information for biological structures reconstruction using Multiple-Angle Total Internal Reflection Fluorescence Microscopy," in International Symposium on Biomedical Imaging (ISBI). IEEE, 2014, pp. 608-611.

[15] J. Boulanger, C. Gueudry, D. Münch, B. Cinquin, P. Paul-Gilloteaux, S. Bardin, C. Guérin, F. Senger, L. Blanchoin, and J. Salamero, "Fast high-resolution 3D total internal reflection fluorescence microscopy by incidence angle scanning and azimuthal averaging," Proceedings of the National Academy of Sciences, vol. 111, no. 48, 2014.

[16] Daniel R Stabley, Thomas Oh, Sanford M Simon, Alexa L Mattheyses, and Khalid Salaita, "Real-time fluorescence imaging with $20 \mathrm{~nm}$ axial resolution," Nature communications, vol. 6, pp. 8307, 2015.

[17] Marcelina Cardoso Dos Santos, Régis Déturche, Cyrille Vézy, and Rodolphe Jaffiol, "Topography of cells revealed by variable-angle total internal reflection fluorescence microscopy," Biophysical journal, vol. 111, no. 6, pp. 1316-1327, 2016.
[18] Luhong Jin, Jian Wu, Peng Xiu, Jiannan Fan, Miao Hu, Cuifang Kuang, Yingke Xu, Xiaoxiang Zheng, and Xu Liu, "High-resolution 3d reconstruction of microtubule structures by quantitative multi-angle total internal reflection fluorescence microscopy," Optics Communications, vol. 395, pp. 16-23, 2017.

[19] Cheng Zheng, Guangyuan Zhao, Wenjie Liu, Youhua Chen, Zhimin Zhang, Luhong Jin, Yingke Xu, Cuifang Kuang, and Xu Liu, "Threedimensional super-resolved live cell imaging through polarized multiangle tirf," Optics letters, vol. 43, no. 7, pp. 1423-1426, 2018.

[20] Emmanuel Soubies, Agata Radwanska, Dominique Grall, Laure BlancFéraud, Ellen Van Obberghen-Schilling, and Sébastien Schaub, "Nanometric axial resolution of fibronectin assembly units achieved with an efficient reconstruction approach for multi-angle-tirf microscopy," Scientific Reports, vol. 9, no. 1, 2019.

[21] Stamatios Lefkimmiatis and Michael Unser, "Poisson image reconstruction with hessian schatten-norm regularization," IEEE transactions on image processing, vol. 22, no. 11, pp. 4314-4327, 2013.

[22] Leonid I Rudin, Stanley Osher, and Emad Fatemi, "Nonlinear total variation based noise removal algorithms," Physica D: nonlinear phenomena, vol. 60, no. 1-4, pp. 259-268, 1992.

[23] Patrick L. Combettes and Jean-Christophe Pesquet, Proximal Splitting Methods in Signal Processing, pp. 185-212, Springer New York, New York, NY, 2011.

[24] Emmanuel Soubies, Sur quelques problèmes de reconstruction en imagerie MA-TIRF et en optimisation parcimonieuse par relaxation continue exacte de critères pénalisés en norme-l0, Ph.D. thesis, Université Côte d'Azur, 2016.

[25] Daniel Sage, Thanh-An Pham, Hazen Babcock, Tomas Lukes, Thomas Pengo, Ramraj Velmurugan, Alex Herbert, Anurag Agarwal, Silvia Colabrese, Ann Wheeler, Anna Archetti, Bernd Rieger, Raimund Ober, Guy M. Hagen, Jean-Baptiste Sibarita, Jonas Ries, Ricardo Henriques, Michael Unser, and Seamus Holden, "Super-resolution fight club: A broad assessment of $2 \mathrm{~d} \& 3 \mathrm{~d}$ single-molecule localization microscopy software," bioRxiv, 2018.

[26] M. Unser, E. Soubies, F. Soulez, M. McCann, and L. Donati, "GlobalBioIm: A unifying computational framework for solving inverse problems," in Computational Optical Sensing and Imaging'17. Optical Society of America, 2017. 